\title{
Thyrotropinoma: one tumour, two different clinical presentations.
}

A. Gómez, S. Maraver, M. Molina, I. Cornejo. A. Muñoz, F. Tinahones.

Endocrinology and Nutrition with Nuclear Medicine Department. Virgen de la Victoria Universitary Hospital. Málaga. Spain

\section{INTRODUCTION}

Thyrotropin-secreting pituitary tumors are $<1 \%$ of pituitary adenomas. Clinical manifestations are similar to other forms of hyperthyroidism. Most of them are diagnosed as macroadenoma (microadenomas $<10 \%$ ).

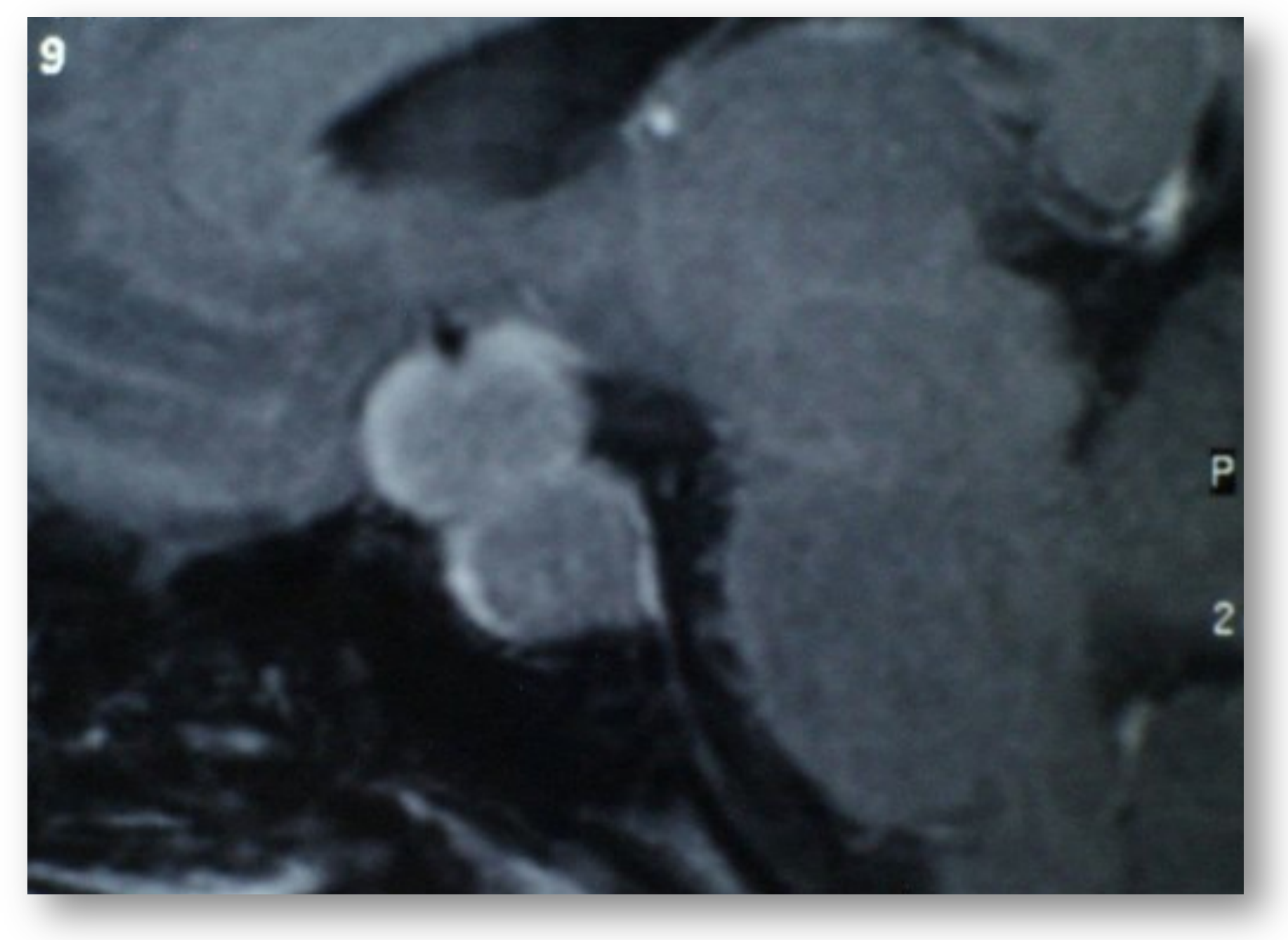

\section{CASE 1}

48 years old woman:

- Menopause presented 6 years ago. Consulting in 2009 for weight loss and palpitations.

- Elastic goiter and distal tremor.

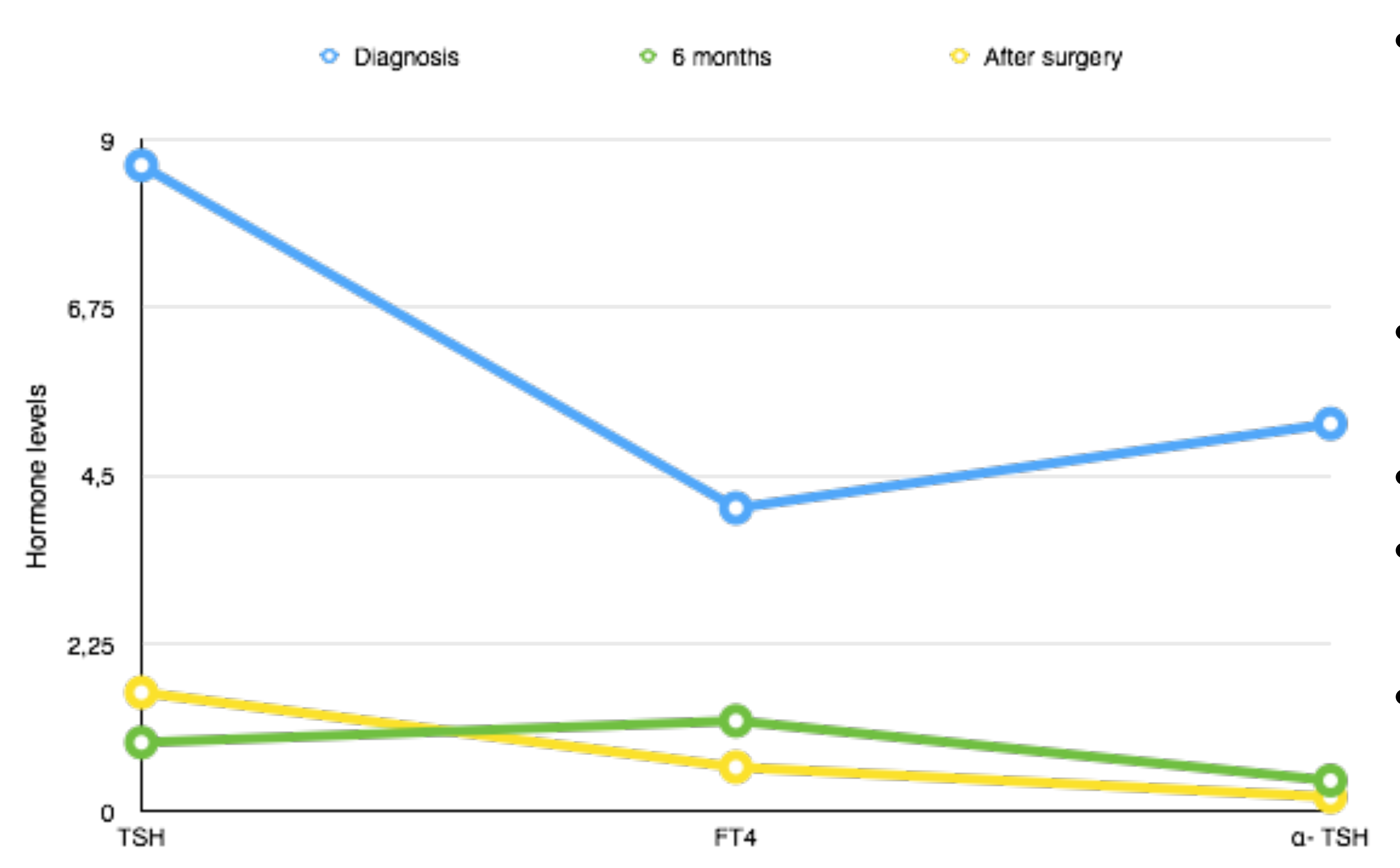

- Blood test: TSH 8.66mcUl/ml (0.2-4.2), FT4 4.7ng/dl (0.7-1.55), FT3 10pg/ml (2-4), $\alpha$-TSH 5.2UI/L (0.02-0.9), negative antithyroid antibodies, FSH $10.2 \mathrm{mUI} / \mathrm{ml}, \mathrm{LH} 4.7 \mathrm{mUI} / \mathrm{ml}$, estradiol $28 \mathrm{pg} / \mathrm{ml}$ and the rest of pituitary hormones in normal range.

- Pituitary MRI: macroadenoma $(2 \mathrm{~cm})$ with suprasellar invasion, contacting optic chiasm.

- Campimetry: normal.

- Treatment was started: propanolol $30 \mathrm{mg} /$ day and octreotide $20 \mathrm{mg} / 28$ days.

- Six months after: clinical improvement and restoration of menstruation, TSH 0.93, FT4 1.22, $\alpha$-TSH 0.42 and slight decrease in tumour size, respecting the optic chiasm (MRI).

- Transsphenoidal surgery in April $2010 \rightarrow$ immunohistochemistry was positive to TSH-GH.

CASE 2

- Immediate postoperative results: TSH 0.04, FT4 1.1. 8 weeks after: panhypopituitarism (TSH 1.6, FT4 0.6, $\alpha$-TSH 0.2).

- After 6 months, patient is stable with sustitutive treatment and there's no tumour in MRI.

49 years old woman:

- History of complete hysterectomy, consulting in 2008 for TSH 8, FT4 2.99 and positive antithyroid antibodies in a blood test.

- She presented irritability and irregular goiter.

- Pituitary MRI: microadenoma $(0.6 \mathrm{~cm})$.

- Blood $\alpha$-TSH 3.2.

- She started octeotride $20 \mathrm{mg} / 28$ days.

- After 6 months $\rightarrow$ TSH 1.7, FT4 1.08 and pituitary lesson was stable.

- Transsphenoidal surgery in 2011 with immunohistochemistry positive to TSH.

- 8 weeks postsurgery: TSH 2.3, T4 0.88, $\alpha$-TSH 0.34.

- 6 months after, thyroid axis is normal and there's no tumour in MRI.

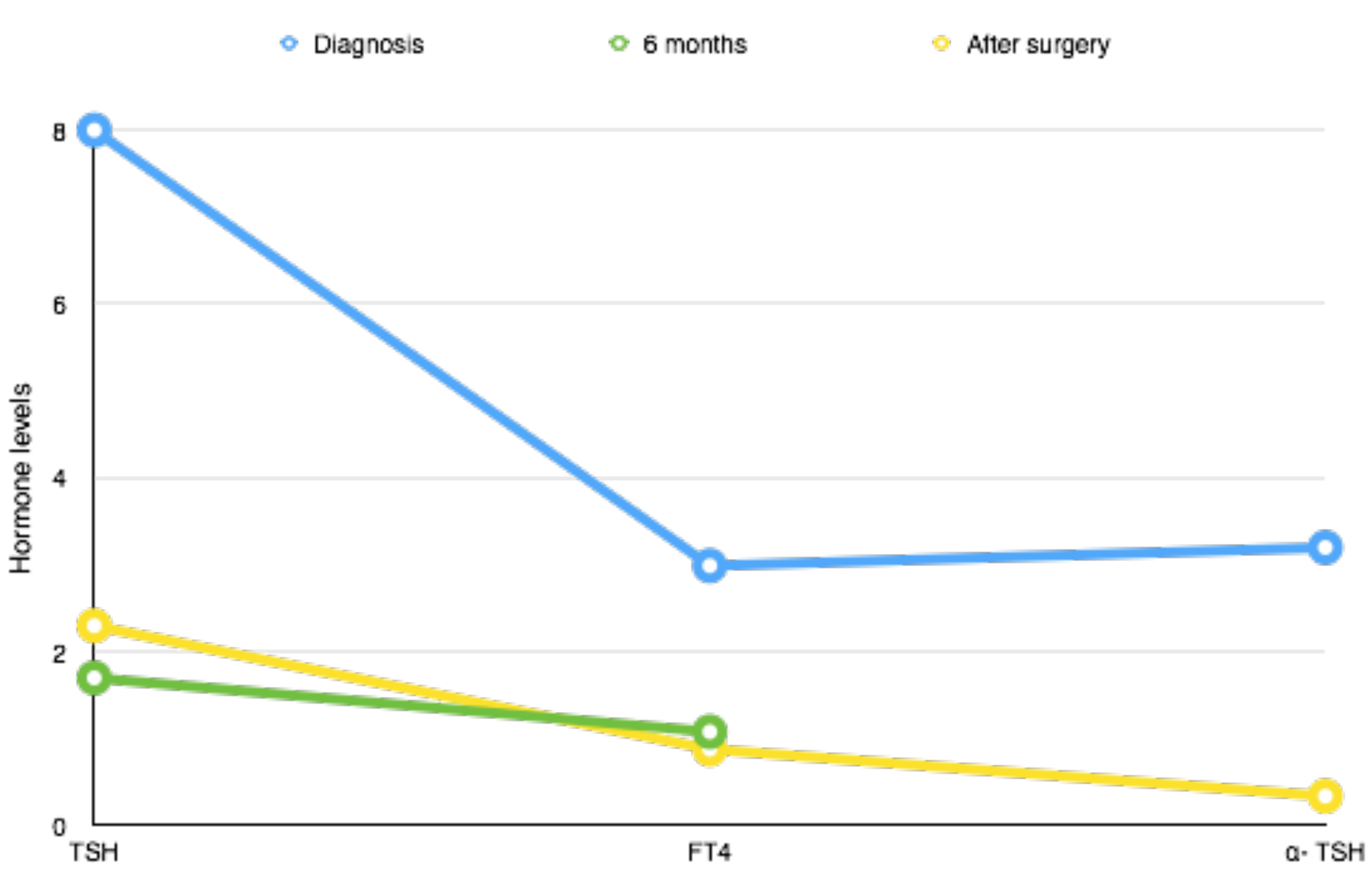

\section{CONCLUSIONS}

We present two different clinical forms of this tumor type, at initial diagnosis and in their postoperative evolution, probably related to tumor size.

However in both cases we observed clinical and analytical response to early medical treatment with somatostatin analogues. 